5. - Harmonic analysis of the classical domains in the study of analytic functions of several complex variables, mimeographed notes, about 1956.

6. J. Mitchell, The kernel function in the geometry of matrices, Duke Math. J. vol. 19 (1952) pp. 575-584.

7. K. Morita, On the kernel functions of symmetric domains, Science Reports of the Tokyo Kyoiku Daigaku, Section A vol. 5 (1956) pp. 190-212.

8. C. L. Siegel, Analytic functions of several complex variables, Notes by P. T. Bateman, Institute for Advanced Study, Princeton, 1948-1949.

The Pennsylvania State University

\title{
A COUNTABLE INTERPOLATION PROBLEM
}

\section{Z. A. MELZAK}

1. Let $\mathfrak{F}$ be the set of all order-preserving homeomorphisms of $I=[0,1]$ onto itself. $\mathcal{H}$ is a metric space in the uniform metric $\rho$ :

$$
\rho\left(f_{1}, f_{2}\right)=\max _{I}\left|f_{1}(x)-f_{2}(x)\right|, \quad f_{1}, f_{2} \in \mathcal{F C} .
$$

Franklin [1] has proved the following theorem: (A) Let $A$ and $B$ be two countable sets, each dense on $I$. Then the set of analytic $f \in \mathcal{H}$, such that $f(A)=B$, is dense in $\mathcal{H}$.

It follows from (A) and from its extension in [2] that there exist nontrivial analytic functions $f \in \mathcal{H}$, such that $f(x)$ is transcendental for each transcendental $x \in I$, and for each algebraic $x \in I, x$ and $f(x)$ are algebraic and of the same degree.

In this note, without using either of these results, we prove a similar but complementary statement by means of Baire's Category Theorem.

THEOREM 1. Let $\mathfrak{K}_{\alpha}, \alpha>2$, be the subset of $\mathfrak{H C}$ consisting of all functions $f \in \mathcal{H}$, whose values are either rational or transcendental and approximable to degree $>\alpha$, for each algebraic $x \in I$. Then $\mathcal{K}_{\alpha}$ is a dense $G_{\delta}$-set of second category in $\mathcal{H}$.

2. Since $\mathcal{F}$ is not complete in $\rho$, we first remetrize it. Let

$$
\sigma\left(f_{1}, f_{2}\right)=\rho\left(f_{1}, f_{2}\right)+\rho\left(f_{1}^{-1}, f_{2}^{-1}\right), \quad f_{1}, f_{2} \in \mathfrak{H} .
$$

Lemma 1. He is complete in the $\sigma$-metric.

Let $\mathcal{F}=I^{I}$ be the set of all continuous maps from $I$ into $I$, then $\mathcal{F}$ is complete in $\rho$. Let $\left\{f_{n}\right\}, n=1,2, \cdots$, be a $\sigma$-Cauchy sequence in FC. Then $\left\{f_{n}\right\}$ is also a $\rho$-Cauchy sequence in $\mathcal{F}$, therefore $f_{n} \rightarrow f$,

Received by the editors May 8, 1959 and, in revised form, May 25, 1959. 
$f \in \mathcal{F}$. Similarly, $\left\{f_{n}^{-1}\right\}$ is also a $\rho$-Cauchy sequence in $\mathcal{F}$, therefore $f_{n}^{-1} \rightarrow h, h \in \mathcal{F}$. Finally, $f[h(x)]=h[f(x)]=x$, therefore $f \in \mathcal{F}$ and $h=f^{-1} \in \mathcal{F}$. Hence $f \in \mathscr{F}$ and $\mathcal{H C}$ is complete.

Henceforth we consider $\mathfrak{H C}$ in the $\sigma$-metric only.

Lemma 2. Let $G$ be an open interval on a line, with rational endpoints. Let $A=\left\{a_{n}\right\}, n=1,2, \cdots$, be the sequence of all rational members of $G$, and put $a_{n}=p_{n} / q_{n}$ where $p_{n}$ and $q_{n}$ are rational integers, $q_{n}>0$, and g.c.d. $\left(p_{n}, q_{n}\right)=1$. Let $\alpha>2$ and $\epsilon>0$. Put

$$
\begin{array}{ll}
A(\epsilon, \alpha)=\bigcup_{n=1}^{\infty}\left(p_{n} / q_{n}-\epsilon / q_{n}^{\alpha}, p_{n} / q_{n}+\epsilon / q_{n}^{\alpha}\right), & 0 \notin G, \\
A(\epsilon, \alpha)=(-\epsilon, \epsilon) \cup \bigcup_{n=1}^{\infty}\left(p_{n} / q_{n}-\epsilon / q_{n}^{\alpha}, p_{n} / q_{n}+\epsilon / q_{n}^{\alpha}\right), & 0 \in G .
\end{array}
$$

Let $\left\{\epsilon_{m}\right\}, m=1,2, \cdots$, be a sequence of positive numbers decreasing steadily to 0 . Let

$$
A_{\alpha}=\bigcap_{m=1}^{\infty} A\left(\epsilon_{m}, \alpha\right) \text {. }
$$

Then $x \in A_{\alpha}$ if and only if either (1) $x \in A$ or (2) $x \in G, x$ is a transcendental number approximable to degree $>\alpha$.

It is clear that $A \subset A_{\alpha}$. Let $y \in G$ be a real number approximable to degree $d>\alpha$. By the definition of this concept $[3 ; 4]$ this means that the equation

$$
|y-p / q|<1 / q^{d}
$$

has infinitely many solutions in rational integers $p, q$, g.c.d. $(p, q)=1$. This implies that $y \in A\left(\epsilon_{m}, \alpha\right)$ for every $m$, and so $y \in A_{\alpha}$. Since $d>2$, $y$ is transcendental by Roth's theorem [5].

Suppose now that $y \in A_{\alpha}, y$ irrational. By the definition of $A_{\alpha}$ this means that the inequality

$$
p / q-\epsilon / q^{\alpha}<y<p / q+\epsilon / q^{\alpha}
$$

is satisfied in rational integers $p, q$, g.c.d. $(p, q)=1$, for arbitrarily small $\epsilon$. Since $y$ is irrational there are infinitely many distinct such solutions. This shows that $y$ is approximable to degree $>\alpha$ and therefore transcendental.

3. We now prove Theorem 1 .

Let $R=\left\{r_{n}\right\}, B=\left\{b_{n}\right\}, n=1,2, \cdots$, be the sets of all rational and all algebraic numbers in $(0,1)$ respectively, taken in the above enumerations. Sets $R\left(\epsilon_{m}, \alpha\right)$ are defined as in (3). Put 


$$
\mathfrak{u}_{n, m}=\left\{f \mid f \in \mathfrak{K}, f\left(b_{n}\right) \in R\left(\epsilon_{m}, \alpha\right)\right\} .
$$

Since $R\left(\epsilon_{m}, \alpha\right)$ is an open set dense on $I$, it follows that $\mathfrak{u}_{n, m}$ is, for each $n, m$, an open set dense in $\mathcal{H}$. By Baire's Theorem [6] the set

$$
\mathcal{U}=\bigcap_{n, m=1}^{\infty} \mathcal{U}_{n, m}
$$

is therefore a dense $G_{\delta}$-set of second category in $\mathfrak{H}$.

Since $f \in \mathcal{U}$ if and only if

$$
f\left(b_{n}\right) \in \bigcap_{m=1}^{\infty} R\left(\epsilon_{m}, \alpha\right), \quad n=1,2, \cdots
$$

it follows from Lemma 2 that $f \in \mathcal{U}$ if and only if for each $n f\left(b_{n}\right)$ is either rational or transcendental and approximable to degree $>\alpha$. Therefore $\mathfrak{U}=\mathscr{K}_{\alpha}$ and the theorem is proved.

CoRollary. Theorem 1 remains true if "approximable to degree $>\alpha$ " is replaced by "a Liouville number."

A number $x$ is a Liouville number $[3 ; 4]$ if it is approximable to any degree. To prove the corollary it suffices to take a sequence $\left\{\alpha_{m}\right\}, m=1,2, \cdots$, of real numbers increasing steadily to infinity and with $\alpha_{1}>2$. We then consider the set

$$
\mathscr{K}_{\infty}=\bigcap_{m=1}^{\infty} \mathcal{K}_{\alpha_{m}},
$$

which is a dense $G_{\delta}$-set of second category in $\mathfrak{H C}$ since each $\mathfrak{K}_{\alpha_{m}}$ is such a set.

\section{REFERENCES}

1. P. Franklin, Analytic transformations of linear everywhere dense point-sets, Trans. Amer. Math. Soc. vol. 27 (1925) pp. 91-100.

2. Z. A. Melzak, Existence of certain analytic homeomorphisms, Bull. Canad. Math. Soc. vol. 2 (1959) pp. 71-75.

3. W. J. Leveque, Topics in number theory, vol. 2, Addison-Wesley, 1956.

4. T. Schneider, Einfuehrung in die transzendenten Zahlen, Springer, 1957.

5. K. F. Roth, Rational approximations to algebraic numbers, Mathematika vol. 2 (1955) pp. 1-20. 1948.

6. W. Hurewicz and H. Wallman, Dimension theory, Princeton University Press,

McGill University 